\title{
COMMENTS ON NOTES OF STANOJEVIĆ ET AL.
}

\author{
MAHLON M. DAY
}

\begin{abstract}
Some further inequalities which characterize inner-product spaces among normed linear spaces are derived by averaging characters of locally compact groups.
\end{abstract}

1. Comparing results in Penico and Stanojević (Proc. Amer. Math. Soc. 79 (1980), 427-430) with the Schoenberg characterization:

$$
\begin{aligned}
& (S, \sim) \text { For all } x, y \text { in } S, 2^{-1}\left(\|x+y\|^{2}+\|x-y\|^{2}\right) \sim 2 \text {, in words: } \\
& \text { the average of }\|x+z y\|^{2} \text { over the group }\{1,-1\} \sim 2,
\end{aligned}
$$

of inner-product spaces (see Day, Normed linear spaces, Chapter VII, §3, for a general view of such characterizations) suggested that a modification of the proof of the Dvoretsky-Rogers Theorem (op. cit., p. 80, Lemma 1) would prove a family of "averaging" characterizations of inner-product spaces. For notation, $\Gamma$ is the circle group in the complex plane, $S$ is the unit sphere $\{x \mid\|x\|=1\}$ in a normed space $N$, and $\sim$ means one of the relations $<,=$, or $>$ but the same one for all $x$ and $y$ in $S$.

TheOREM A. Let $G \neq 1$ be a closed subgroup of the circle group $\Gamma$ and let $H$ be Haar measure on $G$ normalized so that $H(G)=1$. Let

$$
\Psi_{G}(x, y)=\int_{G}\|x+z y\|^{2} d H(z) .
$$

Then a complex-linear normed space $N$ is an inner-product space if and only if

$$
\left(\Psi_{G}, \sim\right) \quad \text { for all } x, y \text { in } S, \Psi_{G}(x, y) \sim 2 .
$$

The note of Stanojević and Suchanek (Proc. Amer. Math. Soc. 81 (1981), 101-103) gives a more general result. Their paper does not, however, suggest the finite examples which are prominent in Theorem $A$ because the only closed proper subgroups of $\Gamma$ are the groups $G_{k}$ of $k$ th roots of unity. If $\theta$ is a principal $k$ th root of unity, then $N$ is an inner-product space if and only if for each (or for one) $k>2$

$$
\left(\Psi_{k}, \sim\right) \quad \text { for each } x \text { and } y \text { in } S, k^{-1} \sum_{p<k}\left\|x+\theta^{p} y\right\|^{2} \sim 2 .
$$

The case $k=2$ is $(S, \sim)$. The case $k=3$ already gives an unfamiliar condition

$$
\left(\|x+y\|^{2}+\|x+\omega y\|^{2}+\left\|x+\omega^{2} y\right\|^{2}\right) / 3 \sim 2,
$$

where, of course, $\omega^{3}=1$.

Received by the editors April 23, 1980.

AMS (MOS) subject classifications (1970). Primary $46 \mathrm{Cl0}$. 
A result similar to Theorem $\mathbf{A}$ gives an averaging analog of the condition of Penico and Stanojević; replace $\Psi_{G}$ above by

$$
\Phi_{G}(x, y)=\int_{G}\|(\operatorname{Re}(z)) x+(\operatorname{Im}(z)) y\|^{2} d H(z) .
$$

One can make a corresponding analog of the theorem of Stanojević and Suchanek by replacing their integrand by $\|(\operatorname{Re}(\gamma(g))) x+(\operatorname{Im}(\gamma(g))) y\|^{2}$.

2. Von Neumann (Trans. Amer. Math. Soc. 36 (1934), p. 445) pointed out that the space $A P(G)$ of almost periodic functions on an arbitrary group $G$ is a natural generalization of the space of continuous functions on a compact group. One aspect of this is the existence on $A P(G)$ of a unique invariant mean $\mathrm{m}$, which specializes to a normalized Haar measure when $G$ is compact. Since each character of $G$ is almost periodic, this point of view allows a generalization of the theorem of Stanojevic and Suchanek sufficiently broad to allow as a corollary the generalization of Theorem $\mathbf{A}$ to all subgroups of $\Gamma$.

TheOREM B. A complex-linear space $N$ is an inner-product space if there exists a topological group $G$ and a continuous character $\gamma \neq 1$ of $G$ such that

$$
\left(\Psi_{\gamma}, \sim\right) \quad \text { for all } x, y \text { in } S, \Psi_{\gamma}(x, y)=m_{g}\left(\|x+\gamma(g) y\|^{2}\right) \sim 2 .
$$

Conversely, if $N$ is an inner-product space, $\left(\Psi_{\gamma},=\right)$ holds for all $G$ and all $\gamma \neq 1$.

The proof is that of Theorem A or of the theorem of Stanojevic and Suchanek.

Since the invariant mean for the almost periodic functions on the integers or on the reals can be calculated as a limit of averages, special cases of Theorem B say that $N$ is an inner-product space if and only if for each (or for one) $z \neq 1$ in $\Gamma$ and for all $x, y$ in $S$

$$
\text { (i) } \quad \lim _{K \rightarrow \infty} K^{-1} \sum_{p<K}\left\|x+z^{p} y\right\|^{2} \sim 2
$$

or

$$
\text { (ii) } \quad \lim _{K \rightarrow \infty}(2 K)^{-1} \int_{-K}^{K}\|x+\exp (i t) y\|^{2} d t \sim 2 .
$$

